\title{
$\mathrm{K}_{2} \mathrm{GeF}_{6}: \mathrm{Mn}$ 荧光粉的化学共沉淀合成、结构测定与发光性能研究 一个绍一个研究型综合实验
}

\author{
魏灵灵, 焦桓 ${ }^{*}$ \\ 陕西师范大学化学化工学院, 西安 710119
}

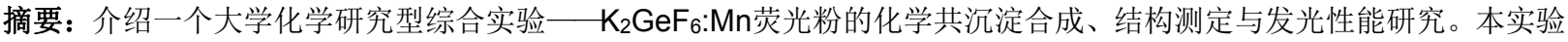
结合氧化还原与化学共沉淀法制备 $\mathrm{K}_{2} \mathrm{GeF}_{6}: \mathrm{Mn}$ 荧光粉, 借助XRD和SEM对粉体进行结构表征, 并利用苂光光谱仪测试 分析其发光性能, 探究激活剂含量和反应温度对粉体结构与性能的影响, 获得可应用于商业白光LED的红色苂光粉。通 过该实验的设计与实施, 使学生在掌握基本实验技能的同时, 能够结合基础理论知识解释实验原理、分析实验现象, 并 进一步提升学生的科学研究思维和综合运用知识的能力。
\end{abstract}

关键词: 研究型综合实验; 化学共沉淀法; 氟化物红色苂光粉; 苂光光谱

中图分类号: G64; O6

\section{Study on Co-precipitation Synthesis, Structure Characterization and Photoluminescent Properties of $\mathrm{K}_{2} \mathrm{GeF}_{6}: \mathrm{Mn}$ Phosphors}

Lingling Wei, Huan Jiao *

School of Chemistry and Chemical Engineering, Shaanxi Normal University, Xi'an 710119, P. R. China.

\begin{abstract}
A research-based comprehensive experiment was introduced: co-precipitation synthesis, structure characterization and photoluminescent properties of $\mathrm{K}_{2} \mathrm{GeF}_{6}: \mathrm{Mn}$ phosphors. Chemical redox and co-precipitation method was employed to synthesize $\mathrm{K}_{2} \mathrm{GeF}_{6}: \mathrm{Mn}$ phosphors. By using XRD, SEM and PL spectra characterization techniques, the effects of $\mathrm{Mn}$ contents and reactive temperatures on the structure and properties of $\mathrm{K}_{2} \mathrm{GeF}_{6}: \mathrm{Mn}$ phosphors were studied. The red phosphors show great potential in LEDs applications. Through this experiment, students can master the basic laboratory skills; interpret the experimental principles with their basic theoretical knowledge, which will further enhance their scientific research ability and comprehensive application of knowledge.
\end{abstract}

Key Words: Research-based comprehensive experiment; Co-precipitation synthesis; Hexafluoride phosphors; Photoluminescence

作为新一代照明光源，白光发光二极管(White Light-Emitting Diode, W-LED)因具有节能环保、 体积小、发热量低、安全可靠、使用寿命长、环境友好、反应速度快、无频闪等诸多优点, 被广泛 用于室内照明、信号指示、汽车照明、农业照明、液晶显示器背光源等领域 ${ }^{[1-3]}$ 。目前商用的白光 LED 主要由 $\mathrm{GaN}$ 蓝色芯片和 $\mathrm{Y}_{3} \mathrm{AlO}_{12}$ : $\mathrm{Ce}$ 黄色荧光粉组合而成。但由于缺少红光成分, 导致这类 LED 器件 的显色指数 Ra 偏低且色温 CCT 偏高, 这些缺点极大地限制了其应用。因此, 在此类 LED 器件中加入 能被蓝光激发的红色荧光粉材料, 可以提高显色指数, 降低色温, 获取应用更广泛的暖白光 $\mathrm{LED}^{[4-6]}$ 。

收稿: 2019-07-28; 录用: 2019-08-15; 网络发表: 2019-09-11

“通讯作者, Email: jiaohuan@snnu.edu.cn

基金资助：陕西师范大学教育教学改革研究项目(GERP-19-42) 
在目前处于研究热点的 $\mathrm{Mn}^{4+}$ 激活的氟化物红色苂光粉中, $\mathrm{Mn}^{4+}$ 离子位于八面体配位的氟化物基 质晶格中, 其发射光谱波峰位置在 $630 \mathrm{~nm}$ 左右, 为集中的线性发射谱线。这种强的窄带光谱特性满 足了 LED 对于理想红色荧光粉的需求。同时, $\mathrm{Mn}^{4+}$ 激活的氟化物荧光粉在紫外和蓝光区都有强且宽 的吸收带, 因此其可以被蓝光芯片有效地激发, 成为极其适用于暖白光 LED 的红色发光材料 ${ }^{[7-9]}$ 。

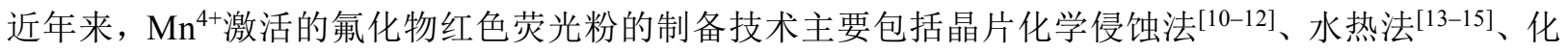
学共沉淀法 ${ }^{[16-18]}$ 。其中, 晶片化学侵蚀法需要以贵重金属或非金属晶片 (Ge、Si、Sn、Ti 与 $\mathrm{Zr}$ 等)作 为反应原料, 制备成本过高且反应时间太长。水热法所使用的反应釜在制备过程中势必会受到高浓 度 HF 酸溶液的腐蚀, 并伴随引发一系列的安全性问题。而化学共沉淀法是指在溶液中含有两种或 多种阳离子, 它们以均相存在于溶液中, 加入沉淀剂后, 可获得具有单一化学组成的沉淀。共沉淀 法操作简单、条件温和, 容易制备具有单一相组成且粒度分布均匀的粉体颗粒。

为了把新的科研成果引入到大学基础实验教学中, 我们设计了 “ $\mathrm{K}_{2} \mathrm{GeF}_{6}: \mathrm{Mn}$ 苂光粉的化学共沉 淀合成、结构与发光性能” 的研究型综合实验。首先结合氧化还原和化学共沉淀法制备配合物 $\mathrm{K}_{2} \mathrm{MnF}_{6}$, 再通过 $\mathrm{HF}$ 酸溶解和化学共沉淀法制备 $\mathrm{K}_{2} \mathrm{GeF}_{6}: \mathrm{Mn}$ 红色荧光粉。通过研究激活剂 $\mathrm{Mn}$ 含量 和反应温度对粉体结构与发光性能的影响, 使学生在掌握材料合成、结构表征及性能测试这些基本 实验技能的同时, 学会运用基础理论知识解释实验原理、分析实验现象, 并进一步提升学生的创新 意识和综合运用知识的能力, 激发学生对科学研究的兴趣 ${ }^{[19]}$ 。

\section{1 实验目的}

(1) 了解制备 $\mathrm{K}_{2} \mathrm{GeF}_{6}: \mathrm{Mn}$ 苂光粉的反应原理与反应条件;

(2) 理解荧光发射原理及红色荧光粉和 LED 发光原理;

(3) 学习化学共沉淀法, 掌握溶解、滴加、沉淀反应、沉淀清洗、过滤、干燥等基本操作;

(4) 学习 X 射线衍射仪、扫描电子显微镜、苂光光谱仪等实验技术;

(5) 掌握 HF 酸的正确使用方法和安全处理措施;

(6) 通过实验过程实施训练学生的科学思维, 培养学生的科研能力和综合运用知识的能力, 结 合 $\mathrm{K}_{2} \mathrm{GeF}_{6}: \mathrm{Mn}$ 红色荧光粉在 LED 器件中的应用拓展, 提升学生的应用型思维。

\section{2 实验原理}

$\mathrm{Mn}$ 属于过渡金属, 过渡金属与常用激活剂稀土离子不同, 它没有最外层全充满的 $5 s^{2} 5 p^{6}$ 电子 层的屏蔽作用。 $\mathrm{Mn}^{4+}$ 作为电子构型为 $3 d^{3}$ 的过渡金属离子, 电子结构独特, $d$ 层电子裸露在最外层。 当 $\mathrm{Mn}^{4+}$ 离子掺杂在无机盐基质中时, 其 $3 d$ 轨道的电子容易受到无机盐晶体场(通常是八面体场)的 影响, 使 $\mathrm{Mn}^{4+}$ 离子的能级擘裂, 从而在紫外或蓝光的激发下可以发生能级跃迁。因此 $\mathrm{Mn}^{4+}$ 离子掺 杂的氟化物荧光粉在蓝光和紫外光区有宽吸收带, 在红光区域有强且窄的发射峰, 能够发出高强度 的红光, 可以作为理想的苂光材料, 应用价值巨大。

在 $\mathrm{K}_{2} \mathrm{GeF}_{6}: \mathrm{Mn}$ 苂光粉中, $\mathrm{K}_{2} \mathrm{GeF}_{6}$ 为无机盐基质, $\mathrm{Mn}^{4+}$ 离子为激活剂。 $\mathrm{K}_{2} \mathrm{GeF}_{6}: \mathrm{Mn}$ 荧光粉的研 究分为合成、结构表征和性能测试三部分。合成方法为化学共沉淀法, 通过对激活剂 Mn 的用量和 反应温度等不同条件下制备出的粉体进行结构与性能分析, 探究最佳合成条件。利用 X 射线粉末衍 射仪(XRD)和扫描电子显微镜(SEM)表征粉体的结构。利用荧光光谱仪对粉体的发光性能进行测试, 表征发光中心的发光波数和发光强度, 分析光致发光机理和发光规律。

合成机理分析如下:

实验所用原料试剂 $\mathrm{KMnO}_{4} 、 \mathrm{KHF}_{2} 、 \mathrm{KF}$ 、和 $\mathrm{GeO}_{2}$ 均易溶于 $\mathrm{HF}$ 酸的水溶液中, 而配合物 $\mathrm{K}_{2} \mathrm{MnF}_{6}$ 和 $\mathrm{K}_{2} \mathrm{GeF}_{6}$ 在 $\mathrm{HF}$ 酸水溶液中的溶解度较小。利用沉淀溶解平衡的原理, 当离子浓度积大于沉淀溶度 积常数时, 就会在总溶液体系中进行合成反应, 析出沉淀。

本实验主要采用二步化学共沉淀法来制备 $\mathrm{K}_{2} \mathrm{GeF}_{6}: \mathrm{Mn}$ 苂光粉。第一步在氧化还原的基础上, 采 
用共沉淀法制备出 $\mathrm{K}_{2} \mathrm{MnF}_{6}$ 配合物; 第二步采用化学共沉淀法制备 $\mathrm{K}_{2} \mathrm{GeF}_{6}: \mathrm{Mn}$ 苂光粉。制备过程中 涉及的主要化学反应方程式有:

$$
\begin{aligned}
& 2 \mathrm{KMnO}_{4}+2 \mathrm{KHF}_{2}+8 \mathrm{HF}+3 \mathrm{H}_{2} \mathrm{O}_{2}=2 \mathrm{~K}_{2} \mathrm{MnF}_{6}+8 \mathrm{H}_{2} \mathrm{O}+3 \mathrm{O}_{2} \uparrow \\
& \mathrm{GeO}_{2}+6 \mathrm{HF}=\mathrm{H}_{2} \mathrm{GeF}_{6}+2 \mathrm{H}_{2} \mathrm{O} \\
& (1-x) \mathrm{H}_{2} \mathrm{GeF}_{6}+x \mathrm{~K}_{2} \mathrm{MnF}_{6}+(2-2 x) \mathrm{KF}=(2-2 x) \mathrm{HF}+\mathrm{K}_{2} \mathrm{Ge}_{1-x} \mathrm{~F}_{6}: \mathrm{Mn}_{x} \downarrow
\end{aligned}
$$

\section{3 实验部分}

\section{1 实验试剂}

$\mathrm{KHF}_{2} 、 \mathrm{KF}$ 、乙醇、丙酮纯度均为 $\mathrm{AR}, \mathrm{KMnO}_{4}(99.5 \%) 、 \mathrm{GeO}_{2}(99.999 \%) 、 \mathrm{HF}$ 酸溶液的质量分 数为 $49 \%, \mathrm{H}_{2} \mathrm{O}_{2}$ 溶液的质量分数为 $30 \%$ 。

\section{2 实验仪器}

实验制备过程中用到的仪器和设备主要有电子天平、电热恒温鼓风干燥箱、 $100 \mathrm{~mL}$ 塑料量杯、 塑料滴管、水槽、磁力加热搅拌器、磁子、吸磁棒、 $5 \mathrm{~mL}$ 移液枪、 $10 \mathrm{~mL}$ 离心管、 $50 \mathrm{~mL}$ 离心管、 保鲜膜、橡皮筋、药匙、和剪刀等。

实验测试仪器如下所示:

(1) 采用日本理学 Mini Flex 600 型 $\mathrm{X}$ 射线粉末衍射仪测试样品的晶体结构, $\mathrm{Cu}$ 靶, $K \alpha$ 辐射, 工作电流为 $50 \mathrm{~mA}$ ，工作电压为 $40 \mathrm{kV}$ ，扫描速度为 $10\left(^{\circ}\right) \cdot \mathrm{min}^{-1}$ ，步长为 $0.02^{\circ}$ 。

(2) 采用美国 FEI 公司 Quanta 200 型环境扫描电子显微镜对样品进行形貌分析。

(3) 采用日本日立公司 F-4600 型苂光光谱仪对荧光粉进行发光性能测试，光源为氙灯。

\section{3 实验过程}

\subsection{1 配合物 $\mathrm{K}_{2} \mathrm{MnF}_{6}$ 的制备}

(1) 在电子天平上称量 $0.45 \mathrm{~g} \mathrm{KMnO}_{4}$ 和 $9.00 \mathrm{~g} \mathrm{KHF}_{2}$ 置于 $100 \mathrm{~mL}$ 塑料量杯中, 加入 $30 \mathrm{~mL} \mathrm{HF}$ 酸溶液, 用保鲜膜盖住量杯口, 并用橡皮筋固定(注意: 塑料量杯中应事先放好磁子, 保鲜膜上用剪 刀预留一小口用来滴加 $\mathrm{H}_{2} \mathrm{O}_{2}$ 溶液)。

(2) 把塑料量杯放在装有冰块的水槽中进行控温, 冰浴下搅拌溶解 $30 \mathrm{~min}$ 。

(3) 溶解完全后, 缓慢滴加 $\mathrm{H}_{2} \mathrm{O}_{2}$ 溶液, 会有大量气泡产生, 同时观察溶液颜色变化(滴加速率控 制为 1 滴/3 $\mathrm{min})$ 。

(4) 当溶液颜色由紫红色变为浅红色时, 继续滴加 1-2 滴 $\mathrm{H}_{2} \mathrm{O}_{2}$ 溶液, 颜色逐渐转变为棕黄色, 并伴随析出大量沉淀。此时, 关闭搅拌器, 迅速取出量杯, 倾斜静置 $2 \mathrm{~min}$, 沉淀沉降后, 揭掉保鲜 膜，用吸磁棒吸出磁子，倒掉上层清液。

(5) 用丙酮洗涤沉淀物 3 次(用量分别为 $20 、 20$ 和 $10 \mathrm{~mL}$ ), 将清洗好的沉淀物即 $\mathrm{K}_{2} \mathrm{MnF}_{6}$ 在 $50^{\circ} \mathrm{C}$ 左右烘干 $2 \mathrm{~h}$ 即可。

\subsection{2 荧光粉 $\mathrm{K}_{2} \mathrm{GeF}_{6}: \mathrm{Mn}$ 的制备}

(1) 在分析天平上称量 $0.55 \mathrm{~g} \mathrm{GeO}_{2}$, 置于 $50 \mathrm{~mL}$ 离心管中(事先放有磁子), 加入 $5 \mathrm{~mL} \mathrm{HF}$ 酸溶 液, 搅拌 $15 \mathrm{~min}$ 使其完全溶解。

(2) 激活剂 $\mathrm{Mn}$ 含量的变化: 按照荧光粉化学计量配比 $\mathrm{K}_{2} \mathrm{Ge}_{1-x} \mathrm{~F}_{6}: \mathrm{Mn}_{x}(x=0.04,0.05,0.06,0.07$, $0.08)$, 称取一定质量的 $\mathrm{K}_{2} \mathrm{MnF}_{6}$ 加入到上述溶液中，继续摚拌混合 $15 \mathrm{~min}$ 备用，反应温度为室温。

(3) 反应温度的影响: 苂光粉化学计量比为 $\mathrm{K}_{2} \mathrm{Ge}_{1-x} \mathrm{~F}_{6}: \mathrm{Mn}_{x}(x=0.06)$, 称取 $0.083 \mathrm{~g} \mathrm{~K}_{2} \mathrm{MnF}_{6}$ 加入 到步骤(1)所得溶液中，离心管分别置于冰水浴中和室温下，继续摚拌混合 $15 \mathrm{~min}$ 备用。

(4) 称量 $0.92 \mathrm{~g} \mathrm{KF}$ ，置于 $10 \mathrm{~mL}$ 离心管中(事先放有磁子)，加入 $3 \mathrm{~mL} \mathrm{HF}$ 酸溶液，摚拌 $30 \mathrm{~min}$ 。 完全溶解后用塑料吸管吸取该溶液滴加到 $\mathrm{GeO}_{2} 、 \mathrm{HF}$ 和 $\mathrm{K}_{2} \mathrm{MnF}_{6}$ 的混合液中, 滴加完成后, 继续摚 拌 $10 \mathrm{~min}$, 此时有大量 $\mathrm{K}_{2} \mathrm{GeF}_{6}: \mathrm{Mn}$ 黄色沉淀生成。

(5) 分别用质量分数为 $25 \%$ 的 $\mathrm{HF}$ 酸溶液、丙酮和无水乙醇洗涤三次, 最后将沉淀物在 $50{ }^{\circ} \mathrm{C}$ 左 
右烘干 2 小时后取出冷却至室温, 称量。

\section{4 结构表征与性能测试}

\section{1 激活剂 $\mathrm{Mn}$ 含量的影响}

图 1 为不同 $\mathrm{Mn}$ 含量的 $\mathrm{K}_{2} \mathrm{Ge}_{1-x} \mathrm{~F}_{6}: \mathrm{Mn}_{x}$ 荧光粉 $(x=0.04,0.05,0.06,0.07,0.08)$ 的 XRD 图。从图中 可以看出, 结合标准衍射卡片 JCPDS No. 73-1531, 当 $x<0.08$ 时, 不同浓度 Mn 含量样品的 XRD 图谱在衍射峰的位置和相对强度上都与标准卡片保持一致, 说明合成产物均为单一相的 $\mathrm{K}_{2} \mathrm{GeF}_{6}: \mathrm{Mn}$,

属于六方晶系中的 $P 3 m 1$ 空间群。当 $\mathrm{Mn}$ 含量为 0.08 时, 结合标准衍射卡片 JCPDS No. 34-0733, 在 XRD 图谱中 $2 \theta=25.5^{\circ}$ 和 $30.8^{\circ}$ 处观察到了 $\mathrm{K}_{2} \mathrm{MnF}_{6}$ 的衍射峰, 说明过多 $\mathrm{Mn}$ 含量的加入虽然没有改 变 $\mathrm{K}_{2} \mathrm{GeF}_{6}$ 基质的晶体结构, 但会超出 $\mathrm{Mn}$ 与 $\mathrm{Ge}$ 在基质中的固溶极限, 从而产生杂质相。

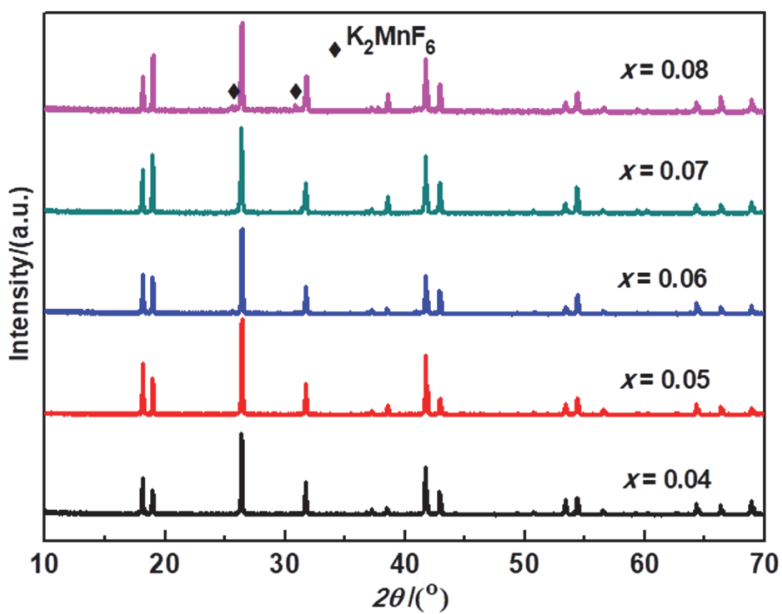

图 $1 \mathrm{~K}_{2} \mathrm{Ge}_{1-x} \mathrm{~F}_{6}: \mathrm{Mn}_{x}(x=004,0.05,0.06,0.07,0.08)$ 红色荧光粉的 XRD 图

图 2(a)为不同 $\mathrm{Mn}$ 含量的 $\mathrm{K}_{2} \mathrm{Ge}_{1-x} \mathrm{~F}_{6}: \mathrm{Mn}_{x}$ 苂光粉 $(x=0.04,0.05,0.06,0.07,0.08)$ 的苂光光谱图。从 图 2(a)左侧的激发光谱图可以看出, 其激发光谱由 $360 \mathrm{~nm}$ 处和 $460 \mathrm{~nm}$ 处的两个吸收宽峰组成, 分 别归属于 $\mathrm{Mn}^{4+}$ 自旋允许的 ${ }^{4} A_{2} \rightarrow{ }^{4} T_{1}$ 和 ${ }^{4} A_{2} \rightarrow{ }^{4} T_{2}$ 的电子跃迁。图 2(a)右侧的发射光谱图是在激发波 长为 $460 \mathrm{~nm}$ 时获得, 从中可以看出, 发射光谱在 590-660 nm 范围内有强的发射峰, 归属于 $\mathrm{Mn}^{4+}$ 的 ${ }^{2} E_{\mathrm{g}} \rightarrow{ }^{4} A_{2}$ 跃迁发射, 其最强发射峰的位置位于 $630 \mathrm{~nm}$ 处, 为典型的红光发射。说明 $\mathrm{K}_{2} \mathrm{GeF}_{6}: \mathrm{Mn}$ 荧 光粉可以很好地与近紫外或蓝光 LED 用 $\mathrm{GaN}$ 芯片结合, 产生高效的红光发射。图 2(b)为 $\mathrm{K}_{2} \mathrm{Ge}_{1-x} \mathrm{~F}_{6}: \mathrm{Mn}_{x}$ 荧光粉的发射光相对强度随 $\mathrm{Mn}$ 含量 $x$ 的变化曲线。从中可以看出荧光强度随着 $x$ 的 增加逐渐增强, 当掺杂浓度为 0.06 时发光强度达到最大, 之后随着 $x$ 的增加荧光强度逐渐减弱, 发 生典型的浓度淬灭现象。由此可知, $\mathrm{Mn}$ 含量的最佳取值为 $x=0.06$ 。

\section{2 反应温度的影响}

图 3 为 $\mathrm{Mn}$ 含量确定为 $x=0.06$ 时, 在不同反应温度下合成的 $\mathrm{K}_{2} \mathrm{GeF}_{6}: \mathrm{Mn}$ 粉体的结构与发光性 能图。从图 3(a)XRD 图谱中可以看出, 在两个不同温度下所合成的粉体均为纯相的 $\mathrm{K}_{2} \mathrm{GeF}_{6}: \mathrm{Mn}_{\text {。结 }}$ 合 SEM 图 3(b) 与图 3(c) 可知, $\mathrm{K}_{2} \mathrm{GeF}_{6}: \mathrm{Mn}$ 为片状六边形形貌, 当反应温度为室温时, 粉体尺寸较为 均匀, 六边形粉体的颗粒大小在 30-50 $\mu \mathrm{m}$ 范围。图 3(d)为 $\mathrm{Mn}$ 含量确定为 $x=0.06$ 时, 在不同反应 温度下合成的 $\mathrm{K}_{2} \mathrm{GeF}_{6}: \mathrm{Mn}$ 粉体的荧光光谱图, 从中可以看出, 当反应温度为室温时, 合成的粉体其 吸收强度和发光强度高, 具有较好的发光性能。由此可知, 采用化学共沉淀法合成 $\mathrm{K}_{2} \mathrm{GeF}_{6}: \mathrm{Mn}$ 荧光 粉的适宜温度为室温。 

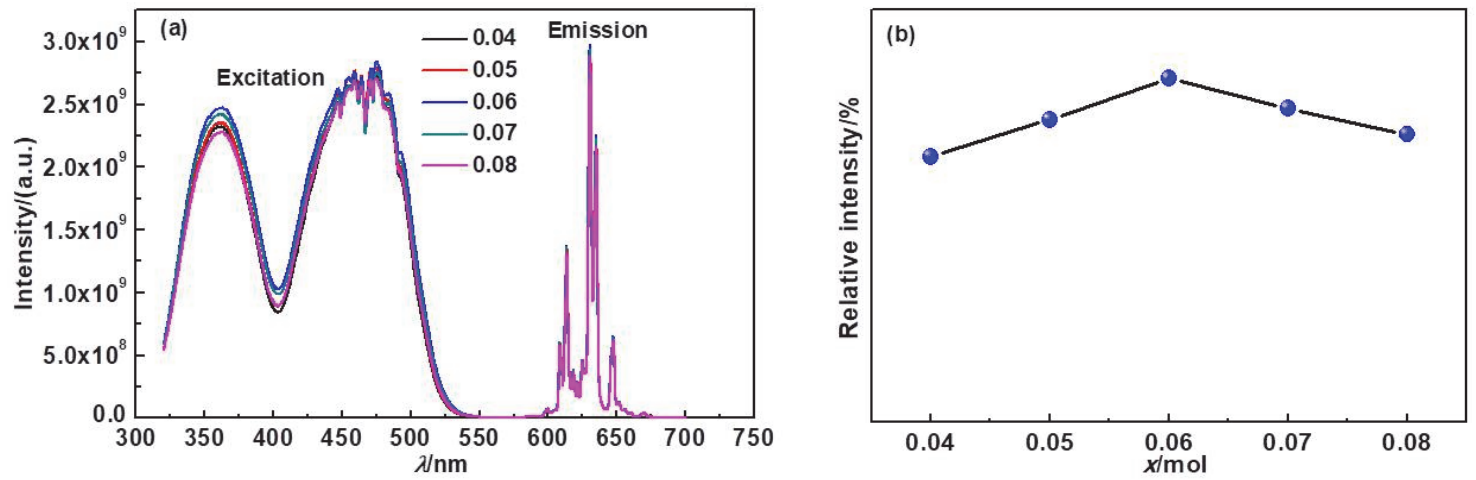

图 $2 \mathrm{~K}_{2} \mathrm{Ge}_{1-x} \mathrm{~F}_{6}: \mathrm{Mn}_{x}(x=004,0.05,0.06,0.07,0.08)$ 红色荧光粉的苂光性能图

(a) 苂光光谱; (b) 发射光相对强度随 Mn 含量 $x$ 的变化曲线

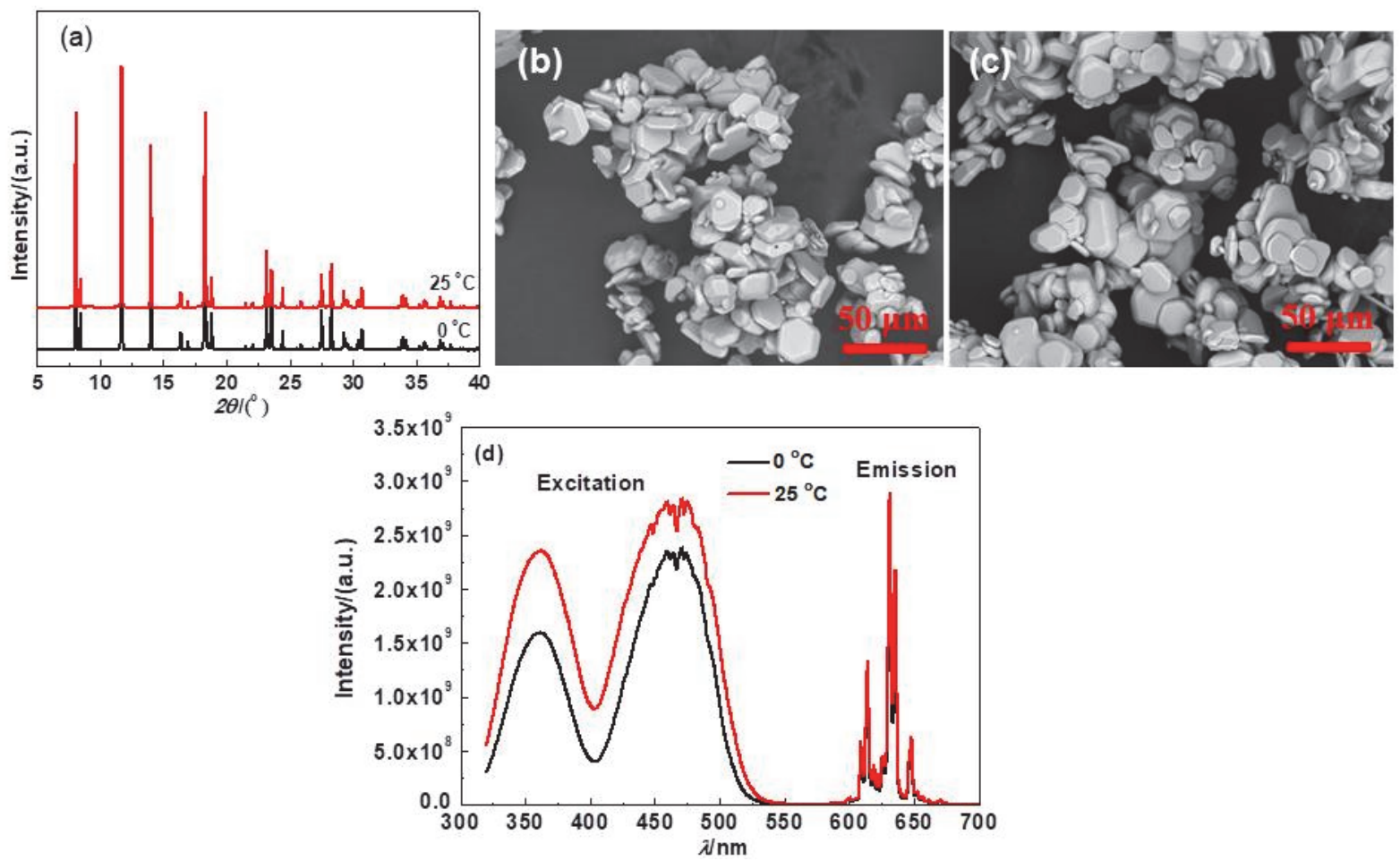

图 $3 \mathrm{Mn}$ 含量确定为 $x=0.06$ 时, 在不同反应温度下合成的 $\mathrm{K}_{2} \mathrm{GeF}_{6}: \mathrm{Mn}$ 苂光粉的结构与发光性能图

(a) XRD 图; (b) SEM 图: $0{ }^{\circ} \mathrm{C}$; (c) SEM 图: $25^{\circ} \mathrm{C}$; (d) 苂光光谱图

\section{3 $\mathrm{K}_{2} \mathrm{GeF}_{6}: \mathrm{Mn}$ 苂光粉在 LED 器件中的应用}

图 4(a)和 4 (b)分别为 $\mathrm{K}_{2} \mathrm{GeF}_{6}: \mathrm{Mn}$ 苂光粉在自然光和紫外灯照射下的照片。从图中可以看出通过 化学共沉淀法合成的 $\mathrm{K}_{2} \mathrm{GeF}_{6}: \mathrm{Mn}$ 为黄色粉末，其在紫外灯照射下具有明亮的红光发射。

为了进一步有效评价 $\mathrm{K}_{2} \mathrm{GeF}_{6}: \mathrm{Mn}$ 荧光粉的发光特性, 我们使用蓝色 LED 芯片作为激发光源, 结合商用的 $\mathrm{Y}_{3} \mathrm{AlO}_{12}$ : Ce 黄色荧光粉和合成的 $\mathrm{K}_{2} \mathrm{GeF}_{6}: \mathrm{Mn}$ 红色荧光粉制作了一个暖白光 LED。该 LED 器件的光谱图如图 4(c)所示, 其由三部分光谱组成: $450 \mathrm{~nm}$ 附近为蓝色 $\mathrm{GaN}$ 芯片的发射峰, 500$600 \mathrm{~nm}$ 区域为 $\mathrm{Y}_{3} \mathrm{AlO}_{12}$ : $\mathrm{Ce}$ 黄色荧光粉的发射峰, 600-630 nm 处为 $\mathrm{K}_{2} \mathrm{GeF}_{6}: \mathrm{Mn}$ 红色荧光粉的发射 峰。内插图白光 LED 器件呈现出明亮的暖白光, 其显色指数 Ra 为 89 , 色温 CCT 为 $3974 \mathrm{~K}$, 说明 $\mathrm{K}_{2} \mathrm{GeF}_{6}: \mathrm{Mn}$ 可应用在白光 LED 照明中。 

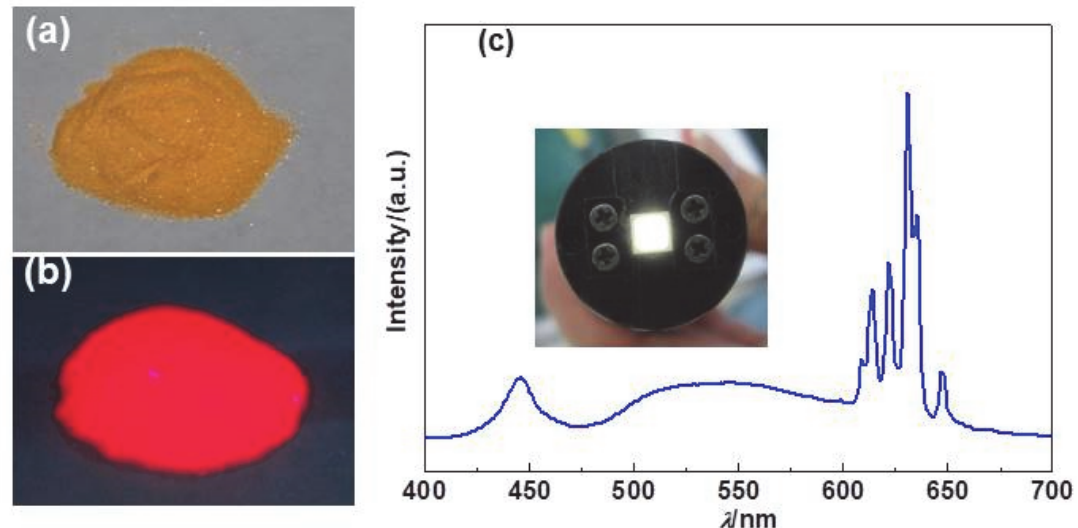

图 $4 \mathrm{~K}_{2} \mathrm{GeF}_{6}: \mathrm{Mn}$ 荧光粉在自然光(a)和紫外灯 (b)照射下的照片及 使用 $\mathrm{K}_{2} \mathrm{GeF}_{6}: \mathrm{Mn}$ 红色荧光粉做成的 LED 器件的光谱图(c)

电子版为彩图

\section{5 注意事项及教学建议}

$\mathrm{HF}$ 酸在使用时必须在通风梪中进行, 且反应容器为塑料制品, 要进行密闭操作。操作人员必须 严格遵守操作规程, 建议佩戴自吸过滤式防毒面具和橡胶耐酸碱手套。HF 酸在领取、放置、使用和 回收过程需要提前进行安全培训, HF 酸废液回收到装有消石灰的专用塑料收集瓶中。本实验过程中 制备出 $1 \mathrm{~g}$ 左右 $\mathrm{K}_{2} \mathrm{GeF}_{6}: \mathrm{Mn}$ 红色荧光粉所用 $\mathrm{HF}$ 酸溶液的体积大概在 $12 \mathrm{~mL}$ 左右, 在事先对学生进 行专门安全培训的前提下, 指导学生严格遵守操作规程, 该实验是处于安全可控范围之内的。若不 慎接触少量 HF 酸后应立即用大量水冲洗, 并在接触部位涂上葡萄糖酸钲凝胶。如果接触范围过广, 医护人员可能会在动脉或周围组织中注射钻盐溶液 ${ }^{[20]}$ 。

由于本实验涉及化学共沉淀制备技术、发光原理、结构表征、性能测试、HF 酸安全防范和规范 处理、应用拓展等环节, 建议以综合实验的形式在大三年级开设。总学时建议为 8 小时：其中实验 准备和安全培训 1 小时, 合成过程 3 小时, 结构表征、性能测试和数据分析 2 小时, LED 拓展应用 1 小时, 讨论交流与反思评价 1 小时。

\section{6 结语}

本实验首先结合氧化还原和化学共沉淀法制备配合物 $\mathrm{K}_{2} \mathrm{MnF}_{6}$, 再通过 $\mathrm{HF}$ 酸溶解和化学共沉淀 法制备 $\mathrm{K}_{2} \mathrm{GeF}_{6}$ : $\mathrm{Mn}$ 红色苂光粉。借助 XRD 和 SEM 对粉体进行结构表征, 并利用荧光光谱仪测试分 析其发光性能, 探究激活剂含量和反应温度对粉体结构与性能的影响, 获得最佳反应条件。白光 LED 器件的封装结果表明该实验制备出的 $\mathrm{K}_{2} \mathrm{GeF}_{6}: \mathrm{Mn}$ 红色荧光粉在商业暖白光领域具有广泛应用前景。

化学共沉淀法是液相反应制备技术中的重要方法, 能级跃迁是离子发光的物理本质。通过该实 验的开设, 使学生在掌握材料合成、结构表征及性能测试这些基本实验技能的同时, 学会运用基础 理论知识解释实验原理、分析实验现象, 并进一步提升学生的创新意识和综合运用知识的能力, 激 发学生对科学研究的兴趣。

[1] Jüstel, T.; Nikel, H.; Ronda, C. Angew. Chem. Int. Ed. 1998, 37, 3084.

[2] 肖志国. 半导体照明发光材料及应用. 北京: 化学工业出版社, 2008 .

[3] Schubert, E. F.; Kim, J. K. Science 2005, 308, 1274. 
[4] Feldmann, C.; Jüstel, T.; Ronda, C. R.; Schmidt, P. J. Adv. Funct. Mater. 2003, 13 (7), 511.

[5] Wang, B.; Lin, H.; Xu, J. ACS Appl. Mater. \& Interfaces 2014, 6 (24), 22905.

[6] Li, J.; Yan, J.; Wen, D.; Khan, W. U.; Shi, J. X.; Wu, M. M.; Su, Q.; Tanner, P. A. J. Mater. Chem. C 2016, 4 (37), 8611.

[7] 周亚运, 王玲燕, 邓婷婷. 中国科学: 技术科学, 2017, 47, 1111.

[8] Adachi, S. J. Lumin. 2018, 197, 119.

[9] Zhou, Y.; Zhang, S.; Wang, X. M.; Jiao, H. Inorg. Chem. 2019, 58 (7), 4412.

[10] Adachi, S.; Takahashi, T. J. Appl. Phys. 2009, 106, 013516

[11] Xu, Y. K.; Adachi, S. J. Electrochem. Soc. 2011, 158, J58.

[12] Kasa, R.; Adachi, S. J. Electrochem. Soc. 2012, 159, J89.

[13] Jiang, X. Y.; Pan, Y. X.; Huang, S. M.; Chen, X. A.; Wang, J. G.; Liu, G. K. J. Mater. Chem. C 2014, $2,2301$.

[14] Lv, L. F.; Jiang, X. Y.; Huang, S. M.; Chen, X. A.; Pan, Y. X. J. Mater. Chem. C 2014, 2, 3879.

[15] Jiang, X. Y.; Chen, Z.; Huang, S. M.; Wang, J. G.; Pan, Y. X. Dalton Trans. 2014, 43, 9414

[16] Wei, L. L.; Lin, C. C.; Fang, M. H.; Jiao, H.; Liu, R. S. J. Mater. Chem. C 2015, 3, 1655.

[17] Huang, L.; Zhu, Y. W.; Zhang, X. J.; Zou, R.; Pan, F. J.; Wang, J.; Wu, M. M. Chem. Mater. 2016, 28 (5), 1495.

[18] Hou, Z. Y.; Tang, X. Y.; Luo, X. F.; Zhou, T. L.; Zhang, L.; Xie, R. J. J. Mater. Chem. C 2018, 6 (11), 2741.

[19] 杨桦. 大学化学, 2018, 33 (10), 79.

[20] 王安桂，孔宪耀，国成波，王玉现，孙兴华. 沂水医专学报, 1984, 6 (2), 243. 